\title{
Médiévales
}

Langues, Textes, Histoire

52 | printemps 2007

Le livre de science, du copiste à l'imprimeur

\section{La science médiévale, du codex à l'imprimé}

\section{Laurence Moulinier-Brogi et Nicolas Weill-Parot}

\section{(2) OpenEdition}

\section{Journals}

Édition électronique

URL : https://journals.openedition.org/medievales/1933

DOI : 10.4000/medievales. 1933

ISSN : 1777-5892

Éditeur

Presses universitaires de Vincennes

Édition imprimée

Date de publication : 1 juin 2007

Pagination : 7-14

ISBN : 978-2-84292-202-3

ISSN : 0751-2708

Référence électronique

Laurence Moulinier-Brogi et Nicolas Weill-Parot, « La science médiévale, du codex à l'imprimé », Médiévales [En ligne], 52 I printemps 2007, mis en ligne le 06 septembre 2009, consulté le 23 avril 2022. URL : http://journals.openedition.org/medievales/1933 ; DOI : https://doi.org/10.4000/ medievales.1933 


\author{
Laurence MOULINIER-BROGI \\ Nicolas WEILL-PAROT
}

\title{
LA SCIENCE MÉDIÉVALE, DU CODEX À L'IMPRIMÉ
}

\author{
«Much has been lost as well as gained \\ in the change from handiwork to machine made things ${ }^{1}$. » \\ Lynn Thorndike
}

Cette remarque de Lynn Thorndike pourrait servir de mémento au médiéviste historien des sciences dès lors qu'il veut utiliser une source. De fait, une grande partie de l'activité scientifique du Moyen Âge n'est encore accessible que dans les manuscrits, dont le catalogage s'avère très inégal selon les fonds ${ }^{2}$. Car l'historien des sciences déchiffre dans les codices et les corpus de manuscrits non seulement un contenu mais aussi les spécificités de leur élaboration et de leur transmission, et est donc confronté à une grande variété : les formes d'écriture, les milieux qui les ont vues naître, les voies qui ont assuré la transmission des textes, ou encore les usages qui en ont été faits, autant de facteurs qui ont pu affecter l'écrit scientifique. Déjà au Moyen Âge, certains auteurs se montraient conscients de cette variété textuelle, tel le médecin Jacques Despars $(†$ 1458) collationnant plusieurs manuscrits du Canon d'Avicenne dont il laissa un imposant Commentaire ${ }^{3}$. Certaines des branches qui constituaient la science médiévale posent ainsi avec force la

1. L. ThORNDIKE, « Manuscript versus Incunabulum », dans Mélanges d'histoire du Moyen Âge dédiés à la mémoire de Louis Halphen, Paris, 1951, p. 693-699.

2. Voir par exemple G. Beaujouan, Manuscrits scientifiques médiévaux de l'Université de Salamanque et de ses "Colegios mayores", Bordeaux, 1962 ; R. CALCOEN, Inventaire des manuscrits scientifiques de la Bibliothèque royale Albert I ${ }^{e r}$, Bruxelles, 2 vol., 1965-1971; J. CoRbett, Catalogue des manuscrits alchimiques latins, I: Manuscrits des bibliothèques publiques de Paris antérieurs au XVII siècle, Bruxelles, 1939 (notice de 97 manuscrits dont 91 pour la Bibliothèque nationale, dans l'ordre des cotes); P. MichELONI, La medicina nei primi tremila codici del fondo Vaticano latino, Rome, 1950 ; L. ScHuBA, Die medizinischen Handschriften der Codices Palatini Latini in der Vatikanischen Bibliothek, Wiesbaden, 1981; ou encore J. Chandelier, L. Moulinier-Brogi, M. Nicoud, « Manuscrits médicaux latins de la Bibliothèque nationale de France: un index des auteurs et des œuvres », Archives d'Histoire doctrinale et littéraire du Moyen Âge, $\mathrm{n}^{\circ}$ 73, 2006, p. 63-163.

3. Cf. D. JacQuart, La Médecine médiévale dans le cadre parisien, Paris, 1998, p. 207-217. 
question de l'importance du manuscrit en raison de la nature protéiforme des textes, comme l'illustre chacune des six études ici rassemblées ${ }^{4}$.

L'article de Jean-Patrice Boudet détruit pour sa part le mythe historiographique selon lequel le collège fondé par Gervais Chrétien aurait été sous Charles V, le «sage roi astrologien », le lieu d'un enseignement majeur de l'astrologie. L'un des instruments de sa démonstration est précisément l'étude de ce que l'on peut reconstituer du fonds manuscrit de ce collège où des ouvrages furent légués, achetés ou copiés. Il montre ainsi comment l'historien peut, à partir de l'étude d'un corpus de manuscrits subsistants, cerner au plus près le projet intellectuel ayant présidé à la création d'une institution, et le milieu qui l'a porté.

C'est un autre mythe qu'Antoine Calvet prend pour cœur de sa réflexion, celui d'un Arnaud de Villeneuve alchimiste sous le nom duquel circulent un grand nombre de textes encore enveloppés d'ombre. Plus que tout autre, le corpus alchimique est difficile à définir, sa tradition manuscrite est labile, propice aux contaminations de toutes sortes. Ces textes échappaient en effet au contrôle de l'université, puisque cette discipline ne figura jamais au nombre des enseignements. Michela Pereira en particulier a tenté de cerner les raisons philosophiques de l'échec de l'intégration de l'alchimie au nombre des disciplines universitaires, qui demeure un fait marquant de l'histoire de cette « science» au tournant des $\mathrm{XIII}^{\mathrm{e}}$ et $\mathrm{XIV}^{\mathrm{e}}$ siècles ${ }^{5}$.

L'anonymat, volontaire ou non ${ }^{6}$, est courant dans d'autres domaines de la science médiévale, mais la tradition textuelle de l'alchimie médiévale est particulièrement affectée par le phénomène de pseudépigraphie ${ }^{7}$. C'est ainsi que la riche personnalité et l'œuvre multiple d'Arnaud de Villeneuve se sont prêtées à la naissance d'une légende faisant du médecin catalan l'auteur d'une transmutation réussie, et l'une des principales figures de l'alchimie occidentale. Des scribes tentèrent même de rassembler en recueil les œuvres de ce Pseudo-Arnaud, mais l'étude de ces textes est d'autant plus ardue que leur nature même a rendu leur tradition éminemment fluctuante. À cette efflorescence contribua sans doute grandement ce qu' on a pu qualifier de «pseudépigraphie accidentelle », à savoir l'attribution d'un traité à un auteur sur la base d'éléments extrinsèques : ainsi l'homonymie, ou la présence d'un texte

4. Le cœur de ce volume est constitué par les communications présentées les 3 et 4 décembre 2004 à Paris lors des journées d'études «Les textes médicaux latins au Moyen Âge : du catalogue de manuscrits à la base de données », organisées par l'Équipe EA 2720 (désormais EA 4116, dirigée par Danielle Jacquart) de l'EPHE, IV ${ }^{\mathrm{e}}$ Section, dans le cadre du projet « Structure de la matière animée face au monde inanimé. $\mathrm{XII}^{\mathrm{e}}-\mathrm{XV}^{\mathrm{e}} \mathrm{s}$. », coordonné par Nicolas Weill-Parot et relevant de l'ACI « Histoire des savoirs » abondée par le CNRS.

5. Voir par exemple à ce sujet C. CRISCIANI, M. PEREIRA, L'arte del sole e della luna. Alchimia e filosofia nel medioevo, Spolète, 1996, chap. II, «L'alchimia nella cultura scolastica », p. 23-53.

6. Songeons par exemple à l'anonymat voulu par l'auteur du Speculum astronomiae : $c f$. A. Paravicini Bagliani, Le Speculum Astronomiae, une énigme? Enquête sur les manuscrits, Florence-Turnhout, 2001.

7. C. Crisciani, M. Pereira, L'arte del sole e della luna, op. cit., p. 77. 
anonyme dans un recueil contenant majoritairement les œuvres d'un auteur donné, ont-elles pu porter à assimiler le premier aux autres, et à le faire circuler par la suite sous le même nom ${ }^{8}$. Il y a donc pour ainsi dire autant de textes qu'il y a de témoins, et le manuscrit se révèle alors plus important encore que le texte.

Mireille Ausécache affronte un problème similaire dans le cadre de son étude sur les antidotaires, ces recueils de médicaments composés dont l'Occident médiéval fut friand, et dont les plus célèbres, tels l'Antidotaire de Nicolas ou le Liber iste attribué à Platearius, virent le jour à Salerne au XII ${ }^{\mathrm{e}}$ siècle. Le Liber iste se présente comme une compilation de plusieurs antidotaires et, de par sa matière, il constituait à son tour un noyau se prêtant à l'amplification, constat qui vaut d'ailleurs aussi pour les recueils de médicaments simples : le Circa instans de Platearius servit ainsi de source principale au Liber simplicium medicinarum, un ouvrage du $\mathrm{XIV}^{\mathrm{e}}$ siècle dont certaines versions contiennent quelque deux cents chapitres de plus que les versions les plus anciennes ${ }^{9}$. En ce qui concerne les antidotaires, les états primitifs du texte sont donc particulièrement difficiles à saisir, de même que la chronologie des compléments et développements ultérieurs.

Le caractère mouvant de ces textes ainsi que leur structure ouverte, propre à l'amplification, sont des caractéristiques de ce champ d'activité qui défient deux étapes essentielles du travail du philolologue, à savoir l'édition et le catalogage. Sans identification sûre, sans Urtext, pas de version légitime, donc a priori pas d'édition plus fiable qu'un manuscrit - difficulté qui se retrouve d'ailleurs pour toutes les traditions d'écriture procédant par énumération de recettes, et pour lesquelles on peut avoir somme toute autant de textes que de copistes. Quant au travail de catalogage, il devient ardu dans une telle configuration. Pourtant, l'élaboration de catalogues est la voie d'accès indispensable à la connaissance d'un corpus et en même temps la clef de sa survie. Dès lors, le choix d'entreprendre de répertorier tel ou tel fonds requiert un effort et un investissement en temps, qui exigent des choix prioritaires : cela explique sans doute que les manuscrits de la science médiévale - domaine d'étude jugé souvent trop spécialisé - n'aient pas toujours été privilégiés. Aussi le choix de cataloguer tel ou tel corpus scientifique relève-t-il le plus souvent de décisions hautement significatives, comme l'illustre le cas des manuscrits scientifiques en catalan.

Le travail de Lluís Cifuentes a pour substrat la diffusion de connaissances scientifiques dans une aire linguistique donnée par le biais du processus de vernacularisation. Il s'intéresse en l'occurrence à l'espace catalan, caractérisé par l'importance, numérique et qualitative, des collections constituées dans cette langue. Ces fonds actuellement conservés en grand nombre à la Bibliothèque nationale de France ont souvent pour origine l'activité, à partir de la

8. R. Halleux, cité ibid., p. 79.

9. Voir Platearius, Le Livre des simples médecines, édition et commentaire par F. AvriL, P. Lieutaghi et G. Malandin, Paris, 1986. 
fin du $\mathrm{XVI}^{\mathrm{e}}$ siècle, de collectionneurs, de même que, par la suite, l'intérêt de savants épris d'histoire de leurs racines, mais les richesses ainsi rassemblées attendaient toujours leur catalogueur. L'article propose donc à la fois une utile mise au point sur l'histoire de la science en catalan, et un précieux instrument de travail sous forme d'inventaire.

Or dans les faits, notre connaissance de l'activité scientifique médiévale repose le plus souvent sur des éditions de textes imprimées à la Renaissance, et est donc en un sens biaisée par le prisme des idées, des intérêts ou des attentes propres à cette période. Les éditeurs du $\mathrm{XVI}^{\mathrm{e}}$ siècle ont en effet procédé tout d'abord à une sélection des auteurs et des œuvres qu'ils voulaient donner à leur presses, et sont en outre intervenus sur les textes en question. Lynn Thorndike en son temps avait ainsi attiré l'attention sur les divergences entre les manuscrits et les éditions de la Chirurgie de Leonardo Bertipaglia $(1421)^{10}$. Un éditeur n'est pas un copiste, et si ce dernier se montre fidèle et scrupuleux à part quelques cacographies involontaires, l'éditeur se place sur un autre registre, doté d'autres ambitions et d'autres moyens : quasiment démiurgique, il est doué du pouvoir de multiplier un texte, qu'il a tout loisir d'adapter à son goût et aux attentes supposées d'un lectorat désormais nombreux, et c'est l'autre corollaire de l'invention de l'imprimerie : un même texte reproductible à l'infini pour tous, contre une version par témoin. Comme l'a souligné Bernard Cerquiglini, l'imprimerie semble en effet avoir porté avec elle la possibilité de réaliser le rêve de la copie fidèle, alors que toute copie est en un sens une altération. L'œuvre du Moyen Âge est une sorte de variable ${ }^{11}$, dans la mesure où, copiée à la main et toujours ouverte, elle se prête à la déformation, à l'intervention ou à la manipulation, et ce jugement semble particulièrement vrai dans différents domaines de la science médiévale.

Manuscrit et incunable, supports complémentaires ou concurrents ${ }^{12}$ ? Le passage à l'imprimé n'a de toute façon rien d'univoque : d'une part la naissance de l'imprimerie ne signe pas l'arrêt de la production manuscrite (songeons à l'humaniste Trithemius qui, conscient d'appartenir, un peu comme Cassiodore, à la fin d'une époque et désireux de sauver pour la postérité certains monumenta, composa en 1492 son Opusculum de laude scriptorum manualium pour exhorter les moines copistes à ne pas se décourager), et d'autre part, l'imprimé n'est pas synonyme de reflet fidèle.

En revanche, l'essor de l'imprimerie s'est accompagné de la mise au point d'une version de référence, voire d'un canon pour certaines œuvres, car c'est également avec le passage du manuscrit à l'imprimé que s'est fait jour le besoin de séparation nette entre écrits apocryphes et authentiques. On crédite

10. Cf. L. Thorndike, «Manuscript versus Incunabulum », loc. cit., p. 694.

11. B. Cerquiglini, Éloge de la variante, Paris, 1989, p. 57.

12. Outre l'article de L. THORNDIKE, « Manuscript versus Incunabulum », on peut consulter sur ce thème G. BAADER, « Handschrift und Inkunabel in der Überlieferung der medizinischen Literatur », dans Medizin im mittelalterlichen Abendland, éd. G. BAADER, G. KEIL, Darmstadt, 1982, p. 359-385. 
de fait les hommes du Moyen Âge d'une certaine indifférence envers les titres d'œuvres ${ }^{13}$, ce que l'on pourrait certes nuancer en évoquant par exemple le médecin Gilles de Corbeil ( $\dagger$ v. 1223) présentant ainsi son De pulsibus : «Tunc ergo sit titulus talis : Incipit liber magistri Aegidii de pulsibus metrice compositus ${ }^{14}$; il semble plus sûr en revanche que la notion d'auteur que l'on avait au Moyen Âge n'est pas la nôtre, et que notre conception du texte est tributaire de siècles d'histoire de l'imprimerie. Avec cette invention qui permit, selon le mot de Bernard Cerquiglini, la conquête de l'«immuable multiple $»^{15}$, les textes furent désormais rattachés à un nom, au prix parfois d'attributions improbables : il suffit de penser à l'éditeur bâlois Hans Kaspar Wolff qui, dans son édition de 1566, imputa l'œuvre connue sous le nom de «Trotula » à un affranchi romain du ${ }^{\text {er }}$ siècle de notre ère, "Eros Juliae », bien que le traité en question fît référence à des auteurs postérieurs ${ }^{16}$ ! La notion d'auteur serait ainsi venue se nicher au cour du texte, désormais vu comme « œuvre de ${ }^{17}$, ce dont témoigne l'apparition progressive de la page de titre telle que nous la connaissons, comportant un nom d'auteur, qui semble acquise au début du XVII ${ }^{\mathrm{e}}$ siècle.

Sebastià Giralt s'intéresse précisément à l'émergence de la notion d'éditeur scientifique - le curador ou curatore du castillan et de l'italien que le français ne sait pas rendre de manière synthétique ${ }^{18}$-, et s'interroge sur la valeur des éditions de la Renaissance pour l'établissement de nos modernes éditions critiques. Son observatoire est constitué par la monumentale entreprise, actuellement en cours à Barcelone, de publication des œuvres médicales d'Arnaud de Villeneuve, connues des spécialistes sous le sigle AVOMO ( A Arnaldi de Villanova Opera Medica Omnia»), et il met notamment en lumière, à propos du jeu complexe entre les tendances du galénisme arabolatin et le courant néo-platonicien à la Renaissance, la dimension idéologique ayant pu sous-tendre le choix d'éditer les écrits d'Arnaud.

À la Renaissance, les manuscrits subsistants d'une œuvre ont parfois été délaissés au profit d'une édition princeps trompeuse, comme le montre l'histoire du Trotula, un ensemble de trois œuvres fondues en un texte unique par son premier éditeur, Georg Kraut, en $1544^{19}$. Et la question du rôle et

13. B. GuENÉE, Histoire et culture historique dans l'Occident médiéval, Paris, 1980, p. 200201.

14. Cité par M. AusÉCACHE, "Le médecin pédagogue au tournant des XII et XIII siècles ", Early Science and Medicine, vol. 3 (1998), p. 187-215.

15. B. CerQuiglini, Éloge de la variante, op. cit., p. 18.

16. Voir les propos liminaires de Wolff, cités par M. H. GreEn, The Trotula. A Medieval Compendium of Women's Medicine, Philadelphie, 2001, p. 206, n. 6: «Erotis medici liberti Iuliae, quem aliqui Trotulam inepte nominant ».

17. B. Cerquiglini, Éloge de la variante, op. cit., p. 57.

18. Pour exprimer la différence entre imprimeur et éditeur scientifique, l'allemand, par exemple, dispose du couple Verlag/Herausgeber et l'anglais de printer/editor.

19. Experimentarius medicinae continens Trotulae curandarum aegritudinum muliebrum ante, in et post partum librum unicum..., Strasbourg, J. Schott, 1544 ; voir à ce sujet F. BERTINI, 
de la responsabilité de l'éditeur scientifique prend d'autant plus de relief que beaucoup de manuscrits médiévaux ayant servi de modèles aux imprimeurs, ont disparu, accidentellement ou non. Il n'était pas rare en effet qu'un éditeur estimât que le manuscrit devenait inutile, avantageusement remplacé par l'édition qu'il donnait, et qu'il s'en débarrassât, comme nous le faisons d'un brouillon. On peut sans doute, avec les auteurs de l'Histoire culturelle de la France, parler de «politique éditoriale» dès les premiers temps de l'imprimerie, et retenir deux cas de figure : soit les imprimeurs adoptaient un point de vue élitiste, en s'adressant délibérément à un public restreint au cercle de leurs amis et des humanistes, soit ils agissaient en hommes d'affaires, en cherchant à offrir une marchandise qui plût au plus grand nombre, et le public déterminait en quelque sorte le programme d'édition ${ }^{20}$.

Un cas parmi tant d'autres, celui du Strasbourgeois Jean Schott, permet d'apprécier différents aspects du problème. On sait par exemple qu'il publia en 1531 une traduction latine de l'œuvre d'Ibn Butlân sous le titre de Tacuinum sanitatis, dans une édition qui, d'après Ernest Wickersheimer, ne respectait pas complètement la disposition des manuscrits ${ }^{21}$. C'est au même homme que l'on doit l'édition princeps du traité naturaliste de Hildegarde de Bingen (†1179) sous le titre de Physica: or ce terme ne figure ni dans les manuscrits qui ont transmis cette œuvre, ni dans les sources qui en font état au Moyen Âge, et il est probable que cette appellation lui fut donnée par l'imprimeur. Il y a de même tout lieu de croire qu'il procéda à des remaniements à partir d'un ou de plusieurs manuscrits comprenant un liber de lapidibus qu'il aurait choisi de ne pas retenir, par souci de suivre le goût du jour : la médecine minérale de Hildegarde s'accompagne de nombreuses formules de conjuration que l'humaniste de Strasbourg tenait peut-être pour d'encombrantes superstitions. Rappelons pour finir que son édition princeps de la Physica figure en tête d'un volume rassemblant trois autres œuvres médicales, attribuées respectivement à Oribase, Théodore et Esculape. Or seul le Liber Esculapii a une source identifiée, l'actuel ms. Bruxelles, Bibliothèque Royale, 13421350 , et l'on ignore, somme toute, quelles sources assigner à trois des quatre écrits contenus dans le recueil publié en $1533^{22}$.

«Trotula, il medico », dans F. Bertini, F. CARdini, C. Leonardi, et alii, Medioevo al femminile, Rome-Bari, 1989, p. 97-119, p. 106-107, et M. H. GreEn, The Trotula, op. cit., p. 59-60.

20. M. Sot, J.-P. Boudet, A. GUerreau-Jalabert, Histoire culturelle de la France, sous la dir. de J.-P. Rioux et J.-F. Sirinelli, t. 1, Le Moyen Âge, dir. M. Sot, Paris, 1997, p. 347.

21. Cf. E. WiCKERSHEIMER, «Les Tacuini sanitatis et leur traduction allemande par Michel Herr », Bibliothèque d'Humanisme et de Renaissance, XII, 1950, p. 85-95, p. 93. Voir aussi Le Taqwim al-Sihha (Tacuini Sanitatis) d'Ibn Butlan: un traité médical du XI ${ }^{e}$ siècle, éd. H. ElKHADEM, Louvain, 1990.

22. L. MoulinIER, Le Manuscrit perdu à Strasbourg. Enquête sur l'œuvre scientifique de Hildegarde, Paris/Saint-Denis, 1995, p. 84. Voir G. SABBAH (dir.), Bibliographie des textes médicaux latins, Antiquité et haut Moyen Âge, Saint-Étienne, 1987, p. 73, 81 et 157. 
Une ombre plus ou moins dense entoure donc certaines éditions de la Renaissance, ce qui rend d'autant plus précieux le témoignage de manuscrits de travail comme ceux de Francesc Argilagues, que Jon Arrizabalaga a récemment exhumés et dont l'étude permet de voir un éditeur à l'œuvre. À l'instar de nombreux congénères, ce Valencien embrassa la voie italienne des études médicales lors d'une peregrinatio academica qui le mena à Sienne, à Pise, et dans différentes villes où il s'adonna à la copie de textes médicaux, ayant choisi de s'installer en Italie plutôt que de rentrer chez lui. Devenu éditeur scientifique à Venise, il publia notamment l'Articella, une collection d'écrits hippocratico-galéniques formant un canon des études de médecine ${ }^{23}$, et le Conciliator du médecin padouan Pietro d'Abano († v. 1315), autant de choix qui témoignent d'un parti pris en faveur de la tradition galénique avicennienne, qu'il cherchait à opposer aux premiers médecins hellénistes. On touche ainsi ici aussi, à travers le cas d'Argilagues, à la dimension idéologique qui put motiver le choix des premiers éditeurs scientifiques.

Si l'on excepte quelques textes, tels le De secretis naturae pseudo-lullien dont la tradition textuelle paraît aussi instable dans les manuscrits que dans les livres imprimés ${ }^{24}$, l'imprimerie a pour ainsi dire effacé les traces de la variance et de la mobilité de la littérature scientifique du Moyen Âge. Il convenait donc de rappeler que la mise au point de l'un démultipliable grâce aux presses impliqua, en amont, tout un travail de sélection, de hiérarchisation, d'interventions sur les textes livrés par les manuscrits, leur faisant subir une ultime manipulation, une dernière toilette, avant d'être fixés en une image indéfiniment reproductible.

Laurence Moulinier-Brogi, Université Paris-VIII, Département d'Histoire, 2, rue de la Liberté, F-93526 Saint-Denis Cedex

Nicolas Weill-Parot, Université Paris-VIII, Département d'Histoire, 2, rue de la Liberté, F-93526 Saint-Denis Cedex

23. Voir entre autres sur ce sujet J. Arrizabalaga, «The Death of a Medieval Text: The Articella and the Early Press », dans R. French, J. Arrizabalaga, A. Cunningham, L. García BAllester (éds.), Medicine from the Black Death to the French disease, Aldershot, 1998, p. 184220.

24. Voir par exemple M. Pereira, The Alchemical Corpus attributed to Raymond Lull, Londres, 1989. 